\title{
Chromospheric structure and activity in solar analogues
}

\author{
M. C. Vieytes ${ }^{1}$, P. J. D. Mauas ${ }^{1}$ and C. C. Cincunegui ${ }^{1}$ \\ ${ }^{1}$ Instituto de Astronomía y Física del Espacio, CC. 67 Suc.28 (1428), Buenos Aires, \\ Argentina. email: mariela@iafe.uba.ar
}

\begin{abstract}
We computed chromospheric models of the Sun as a star and of nine solar analogues. The stars have a wide variety of magnetic activity levels, which allows us to study the differences in atmospheric structures induced by activity.
\end{abstract}

The stars in our sample were chosen with $0.62<\mathrm{B}-\mathrm{V}<0.68$ (the solar $B-V=0.65$ ) and present differences in activity level. The activity indicator is the $\mathrm{S}$ index, which is esentially the flux in the Ca II H and K line cores (Baliunas et al., 1998). Table 1 shows the stellar characteristics. The stellar observations were made at the $2.15 \mathrm{~m}$ telescope of the CASLEO observatory, in the Argentinian Andes (see Cincunegui \& Mauas 2004), and the solar profiles were taken from the FTS atlas (Brault \& Neckel, 1987).

We built a different chromospheric model for each star in the sample, using the code Pandora (Avrett \& Loeser 1992). The atmospheric models were constructed to obtain the best possible match with the Ca II K and $\mathrm{H} \beta$ lines, changing the solar model as little as possible. In some of the stars, we included macroscopic velocity fields to reproduce the assymetries in the line profiles. After the model was finished, we checked it comparing the computed profiles of the $\mathrm{Mg}$ I b and the $\mathrm{Na}$ I D lines with the observations. Our models are shown in Figure 1. Figure 2 shows the observed and calculated profiles for HD 172051 and HD 30495.

Concerning the models for the least active stars, including the Sun, the main change with increasing activity level is in the temperature-minimum region (figure 1a). The chromospheric plateau and the position of the transition region are the same among all these stars. On the other hand, the most active stars show changes all along their atmospheric structures, mainly in the upper chromosphere and in the position of the transition region (figure 1b). These results are in accordance with Pasquini (1992), who studied the Ca II K line for a sample of solar-type stars (G0-G5 spectral type). He showed that, as activity increases, the first changes to appear are in the $K_{1}$, and for larger activity levels also the $K_{3}$ changes. Regarding the macroscopic velocity fields, we can distinguish between the two groups. The least active group has velocities only in the chromospheric plateau, while in the most active stars the temperature-minimum region is affected by the velocities.

To constrain the amount of chromospheric heating required to sustain the atmosphere of the stars, we compute the radiative cooling rate $\phi\left(\mathrm{ergs} \mathrm{cm}^{-3} \mathrm{sec}^{-1}\right)$. Figure 3 shows $\phi_{\text {int }}$, the positive values (which can be considered as the total amount of chromospheric heating needed to sustain the atmosphere) of the cooling-rate integrated from the temperature-minimum up to the height in the transition region where the temperature reaches $10^{4} \mathrm{~K}$, as a function of the $\mathrm{S}$ index. The line shows the linear regression which has a correlation coefficient $r=0.9969$. There is a very good linear relation between them, and therefore S can be used reliably to predict the star's energy requirements from a given activity level. 


$\begin{array}{lcccccc}H D & \text { Spec.type } & B-V & U-B & {[\mathrm{Fe} / \mathrm{H}]} & S_{\text {CTIO }} & S_{M W \max } \\ 189567 & \text { G3 V } & 0.64 & 0.07 & -0.30 & 0.185 & - \\ 197076 & \text { G5 V } & 0.63 & 0.09 & - & - & 0.180 \\ 172051 & \text { G5 V } & 0.63 & 0.17 & - & 0.181 & - \\ 146233 & \text { G2 Va } & 0.65 & 0.16 & 0.05 & - & 0.186 \\ 19467 & \text { G3 V } & 0.65 & 0.15 & - & - & - \\ 20766 & \text { G2.5 V } & 0.64 & 0.08 & -0.22 & 0.245 & - \\ 30495 & \text { G3 V } & 0.64 & 0.13 & -0.13 & 0.286 & 0.363 \\ 1835 & \text { G3 V } & 0.67 & 0.22 & -0.01 & 0.344 & 0.439 \\ 202917 & \text { G5 V } & 0.65 & - & - & 0.743 & -\end{array}$

Table 1. Star characterisitcs (increasing activity level order)
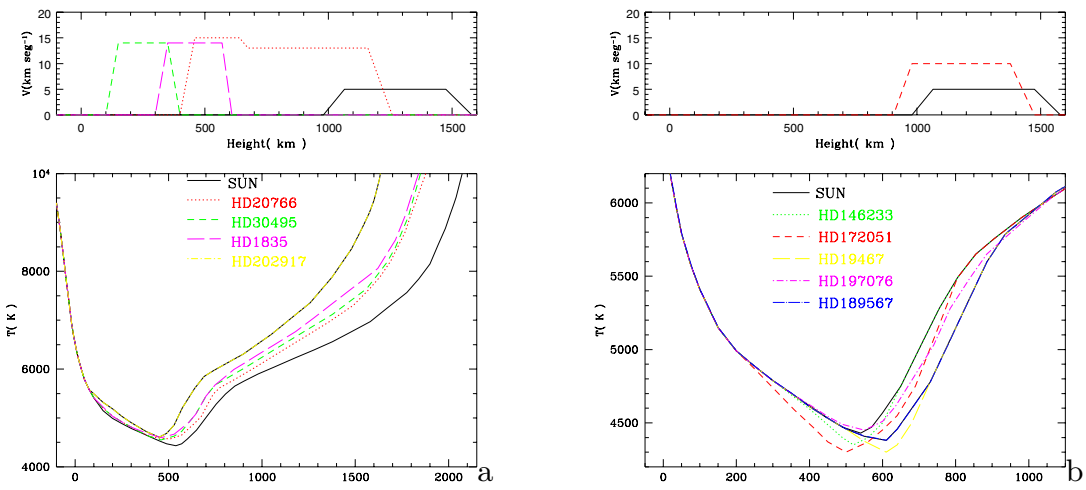

Figure 1. a. Models for the least active stars. b. Models for the most active stars. Top:Macroscopic velocity fields for the corresponding star.
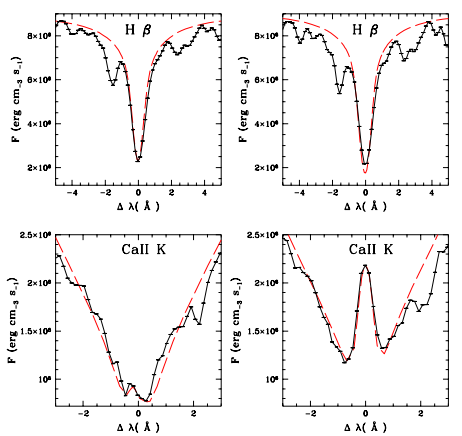

Figure 2. Observed and computed Ca II K and $H \beta$ profiles for HD 172051 (right) and for HD 30495 (left).

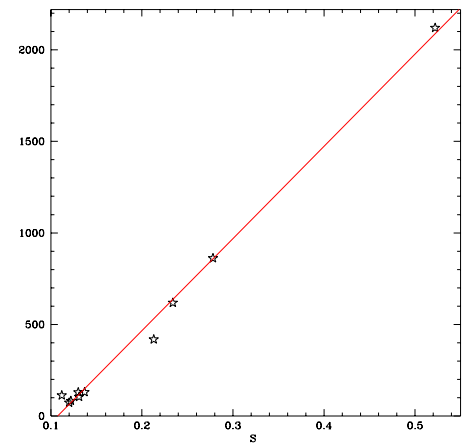

Figure 3. Integrated cooling-rate as a function of the $\mathrm{S}$ index.

\section{References}

Avrett E., Loeser R. 1992 in the 7th Cambridge Workshop on Cool Stars, Stellar Systems and the Sun

Baliunas S., Donahue R., Soon W., Henry G. 1998 Cool Stars, Stellar Systems and the Sun, $A S P$ 154, 153.

Brault \& Neckel 1987 Solar Phys. 184, 421.

C. Cincunegui \& P.J.D. Mauas 2004 A\&SA 414, 699.

Pasquini L. 1992 AËA 266, 347. 